\title{
Using Solvation Free Energy as an Additional Parameter for Corrosion Inhibition Inspection of Organic Compounds in Acid Media: An Evaluation Study
}

\author{
Brahim El Ibrahimi ${ }^{1,2}$ \\ ${ }^{1}$ Faculty of Applied Sciences, IBN ZOHR University, Aït Melloul 86153, Morocco \\ ${ }^{2}$ Team of Physical Chemistry and Environment, Faculty of Sciences, IBN ZOHR University, Agadir 8106, Morocco
}

Correspondence should be addressed to Brahim El Ibrahimi; brahim.elibrahimi@edu.uiz.ac.ma

Received 30 April 2021; Accepted 8 February 2022; Published 28 February 2022

Academic Editor: Qian Chen

Copyright ( $\odot 2022$ Brahim El Ibrahimi. This is an open access article distributed under the Creative Commons Attribution License, which permits unrestricted use, distribution, and reproduction in any medium, provided the original work is properly cited.

The energies of frontiers molecular orbitals and derived electronic parameters are widely used to explain experimentally obtained inhibition effectiveness of several organic inhibitors against metallic corrosion. In this context, the present study aims to reevaluate the use of solvation free energy $\left(\Delta G_{\text {Solv }}\right)$ as an additional parameter for the corrosion inhibition inspection. Three organic molecules set previously reported as corrosion inhibitors in acidic solutions were selected to conduct this in silico study. The solvation free energy in the aqueous phase of chosen inhibitors was determined using DFT-based calculations in combination with integral equation formalism-polarizable continuum solvation model. The acquired data show that $\Delta G_{\text {Solv }}$ can be used together with other known parameters to inspect the inhibition performance of organic inhibitors in acidic media.

\section{Introduction}

The contact of many metals and alloys with acid media during their use (e.g., acid pickling and cleaning) involves corrosion of these materials, which implies a great economic and potential lives loss $[1,2]$. To overcome this undesirable phenomenon, the introduction of corrosion inhibitor compounds into corrosive solutions is known as a practical and attractive method [3, 4]. Many oxygen, nitrogen, and sulfur-containing organic compounds have been reported to act as good corrosion inhibitors for various metallic materials in acid media [5]. Among reported compounds, there are 1,5-benzodiazepin-2-one derivatives [6], 1,2,4-triazolebased compounds [7], and several simple $\alpha$-amino acids [8].

Nowadays, the application of computational modeling approach in the inhibition of corrosion search has been widely reported in the literature [9-11]. Such an approach is applied to understand and to explain observed inhibiting activity at molecular and atomic scale [12]. In this context, various electronic structure parameters characterizing the inhibitor molecule (indicators of reactivity), e.g., LUMO,
HOMO and gap energies, were calculated using density functional theory-based methods, after that they are correlated through simple linear, multilinear, or nonlinear models with the obtained inhibition efficiencies [13-16].

On the other hand, as almost inhibition processes occur in the aqueous solutions, the solvation process of the inhibitor molecule is of interest, which can be estimated via its free energy of solvation $\left(\Delta G_{\text {Solv }}\right)$ [17]. This thermodynamic parameter has been used to evaluate the impact of solvent on the property of molecules in many scientific disciplines, such as pharmaceutical, biological, and chemical sciences [18]. In this regard, the present work aims to evaluate the use of this parameter in the field of corrosion inhibition, i.e., expect the inhibition ability of organic inhibitors largely employed in acid media. This subject has been not sufficiently studied in the literature and is still lacking. From the computational point of view, there are two different theoretical manners to predict $\Delta G_{\text {Solv }}$, namely, implicit and explicit considering solvent molecules. It is well known that continuum solvation models (i.e., implicit approach), such as integral equation formalism-polarizable continuum model (IEF-PCM), are 
TABLE 1: Chosen organic inhibitors data set and corresponding experimental data.

\begin{tabular}{|c|c|c|c|c|c|}
\hline Inhibitor (abbreviation) & Solution & Metal & [Inh.] (mM) & IE $(\%)$ & Ref. \\
\hline 1,2,4-Triazole (TR) & & & & 66 & \\
\hline 3-Amino-1,2,4-triazole (ATR) & $1 \mathrm{M} \mathrm{H}_{2} \mathrm{SO}_{4}$ & Copper & 1 & 71 & {$[6]$} \\
\hline 3,5-Diamino-1,2,4-triazole (DATR) & & & & 73 & \\
\hline 4,7-Dimethyl-1,5-benzodiazepin-2-one (DMBD) & & & & 88 & \\
\hline 3-Phenyl-1,5-benzodiazepin-2-one (PBD) & HCl & Iron & 10 & 90 & [7] \\
\hline 4-Methyl-7-phenyl-1,5-benzodiazepin-2-one (MPBD) & & & & 96 & \\
\hline Asparagine (Asn) & & & & 45 & \\
\hline Glutamine (Gln) & & & & 40 & \\
\hline Alanine (Ala) & & & & 25 & \\
\hline Acide glutamique (Glu) & $1 \mathrm{M} \mathrm{HNO}_{3}$ & Copper & 1 & 38 & {$[8]$} \\
\hline Acide aspartique (Asp) & & & & 28 & \\
\hline Leucine (Leu) & & & & 34 & \\
\hline Threonine (Thr) & & & & 50 & \\
\hline
\end{tabular}

computationally fast than those with the explicit introduction of solvent molecules [19-21].

\section{Computational Details}

2.1. Data Set. Three classes of organic inhibitor molecules are selected randomly to perform this study, namely, 1,2,4-triazole (3 inhibitors), 1,5-benzodiazepin-2-one (3 inhibitors), and $\alpha$-amino acid (7 inhibitors) set. All these compounds have been experimentally tested and reported as corrosion inhibitors in acid media for iron and copper metals [6-8]. To avoid possible differences in the obtained inhibition efficiencies of considered classes, which can be due to how the experimentations were done, the same authors have evaluated each of the above-listed classes of inhibitors. Obtained inhibition effectiveness (IE), as well as prior adopted operating conditions, are summarized in Table 1. The molecular structure of these organic inhibitors is depicted in Figure 1.

2.2. Solvation Free Energy Calculations. The calculation of solvation free energy $\left(\Delta G_{\text {Solv }}\right)$ of chosen organic inhibitors was conducted using DFT/B3LYP method at 6-311++G $(d$, p) basis set $[22,23]$ in combination with the IEF-PCM solvation model by setting water as a solvent [24-27]. As the acidic environment was used in the experimental studies, its effect on the protonation state of inhibitor molecules cannot overlook, for this, the protonated forms of these inhibitors are considered throughout the calculations using Gaussian 09 software. The full optimization of studied molecules was checked by the absence of possible imaginary frequencies [28]. Figure 1 displays the optimized molecular structure of selected organic inhibitors.

\section{Results and Discussion}

There is a large agreement in the literature that the ability of an organic inhibitor to prevent metallic corrosion is a consequence of its adsorption on the metal surface $[29,30]$. However, the adsorption of the inhibitor molecule can occur after its prior release from its solvation shell in the aqueous solution. Consequently, the stronger hydration (i.e., lower $\Delta G_{\text {Solv }}$ values) of an inhibitor molecule can decrease its capacity to adsorb on the metallic surface, which leads to lower protection of metal against corrosion, i.e., lesser inhibition efficiency is obtained [31].

Beginning by the smaller inhibitor set containing three inhibitors per each set, namely, 1,2,4-triazole and 1,5-benzodiazepin-2-one ones, the calculated solvation free energies $\left(\Delta G_{\text {Solv }}\right)$ using IEF-PCM solvation model for the protonated form of 1,2,4-triazole and 1,5-benzodiazepin-2-one inhibitors set are listed in Table 2 . As can be noted in this table, all calculated values indicate that the hydration process of selected organic inhibitors is an exothermic process. The obtained solvation free energy values are ordered as $\mathrm{TR}<\mathrm{ATR}<\mathrm{DATR}$ and $\mathrm{DMBD}<\mathrm{PDB}<\mathrm{MPBD}$ for $1,2,4$ triazole and 1,5-benzodiazepin-2-one inhibitors set, respectively. It is clear that the latter ranking is coherent with the experimentally recorded inhibition effectiveness for both inhibitors classes, in which less $\Delta G_{\text {Solv }}$ value is associated with higher inhibition efficiency. Meaning that the adsorption affinities of DATR and MPBD inhibitors are great than other ones in the same inhibitor set. Hence, better inhibition efficiencies are obtained for DATR and MPBD inhibitors. Furthermore, the computed correlation coefficients $(R)$ between solvation free energy and inhibition efficiency are 0.96 and 0.86 for 1,2,4-triazole and 1,5-benzodiazepin-2-one inhibitor set, respectively, indicating a remarkable proportional $\Delta G_{\text {Solv }} /$ IE relationship. This finding outlines the possibility to employ the $\Delta G_{\text {Solv }}$ energy as a useful parameter to shed more light on the observed inhibition efficiency of these organic inhibitors in acid media.

Evaluating now the $\Delta G_{\text {Solv }} /$ IE relationship on the third inhibitors set, i.e., $\alpha$-amino acid molecules, this larger set includes eight inhibitors, in which their molecular structures are more diverse (see Figure 1). Figure 2 presents the linear correlation curve between calculated $\Delta G_{\text {Solv }}$ and experimentally obtained inhibition efficiency of $\alpha$-amino acids set. As can be noted in this figure, the $\Delta G_{\text {Solv }} / \mathrm{IE}$ relationship is acceptable because the calculated correlation coefficient is 0.70. This means that $\Delta G_{\text {Solv }}$ can explain over $50 \%$ of the obtained experimental inhibition efficiency of this set, which is an interesting achievement. Moreover, it is evident from Figure 2 that the slope of the plotted line (red line in the figure) is positive, which means an increase of the inhibition efficiency of evaluated $\alpha$-amino acid inhibitors by increasing 




FIGURE 1: Optimized molecular structure of selected organic inhibitors in this study using DFT/B3LYP method with 6-311++G $(d, p)$ basis set in the aqueous phase.

TABLE. 2: Calculated solvation free energy $\left(\Delta G_{\text {Solv }}\right)$ and experimentally obtained inhibition efficiency (IE) of 1,2,4-triazole and 1,5-benzodiazepin-2-one inhibitors set.

\begin{tabular}{lccccr}
\hline \multirow{2}{*}{ Inhibitor } & \multicolumn{3}{c}{$1,2,4$-Triazole } & \multicolumn{3}{c}{ 1,5-Benzodiazepin-2-one } \\
& TR & ATR & DATR & DMBD & PDB \\
\hline$\Delta G_{\text {Solv }}\left(\mathrm{Kcal} \mathrm{mol}^{-1}\right)$ & -73.97 & -70.98 & -70.97 & -61.77 & -57.91 \\
IE (\%) & 66 & 71 & 73 & 88 & -56.47 \\
\hline
\end{tabular}



FIGURE 2: Linear correlation curve of $\Delta G_{\text {Solv }}$ with IE of chosen $\alpha$-amino acids in protonated form.

their $\Delta G_{\text {Solv }}$. The obtained data reveal that the solvation free energy can be also served to expect the inhibition effect of a large organic inhibitor set with diversity in their molecular structures.

It is important to underline that the inhibition of corrosion is a complex phenomenon that implies the extrinsic (i.e., experimental conditions, e.g., metal nature, corrosive solution, and temperature) and intrinsic (i.e., inhibitor property) factors to be understood [32]. Under given operational conditions, as presumed to conduct the current study, in addition to the solubility (solvation) of the inhibitors, this phenomenon involves also the electronic/ molecular aspects of the inhibitor molecules and their interfacial interactions with the metallic surface as well. In other words, we can implement the $\Delta G_{\text {Solv }}$ parameter within the known theoretical approach to understand more the noted inhibition trend in acidic media. On the other hand, the energies of frontiers molecular orbitals and derived electronic parameters are commonly used to explain experimentally obtained inhibition effectiveness of several organic inhibitors against metallic corrosion [33]. However, in some cases, it has reported the noncoherence between those parameters and the observed inhibition behaviors, which is due to the complexity of the studied inhibition systems [34-37]. In this context, the use of $\Delta G_{\text {Solv }}$ can also show some exceptional cases in this regard [38].

\section{Conclusion}

The present evaluation study repoints out the possibility to employ the solvation free energy $\left(\Delta G_{\text {Solv }}\right)$ as an intrinsic property of the inhibitor molecule to inspect its inhibition ability against metallic corrosion in acid media. Such a parameter can be implemented within the widely adopted 
theoretical demarche in the literature to understand the observed inhibition trend for a given inhibitor set under fixed extrinsic conditions.

\section{Data Availability}

No additional data were used to support this study.

\section{Conflicts of Interest}

The author declares that he has no conflicts of interest.

\section{References}

[1] G. H. Koch, N. G. Thompson, O. Moghissi, J. H. Payer, and J. Varney, IMPACT (International Measures of Prevention, Application, and Economics of Corrosion Technologies Study), NACE International, Houston, TX, USA, 2016.

[2] M. Abdallah, H. M. Al-Tass, B. A. Al Jahdaly, and A. S. Fouda, "Inhibition properties and adsorption behavior of 5-arylazothiazole derivatives on 1018 carbon steel in $0.5 \mathrm{M} \mathrm{H} 2 \mathrm{SO} 4$ solution," Journal of Molecular Liquids, vol. 216, pp. 590-597, 2016.

[3] L. L. Liao, S. Mo, H. Q. Luo, and N. B. Li, "Corrosion protection for mild steel by extract from the waste of lychee fruit in $\mathrm{HCl}$ solution: experimental and theoretical studies," Journal of Colloid and Interface Science, vol. 520, pp. 41-49, 2018.

[4] R. S. Abdel Hameed and M. Abdallah, "Corrosion inhibition of carbon steel in $1 \mathrm{M}$ hydrochloric acid using some pyrazolo[3,4d]Pyrimidnone derivatives," Protection of Metals and Physical Chemistry of Surfaces, vol. 54, no. 1, pp. 113-121, 2018.

[5] B. El Ibrahimi, A. Jmiai, L. Bazzi, and S. El Issami, "Amino acids and their derivatives as corrosion inhibitors for metals and alloys," Arabian Journal of Chemistry, vol. 13, no. 1, pp. 740-771, 2020.

[6] A. Soumoue, B. E. Ibrahimi, S. E. Issami, and L. Bazzi, "Some triazolic compounds as corrosion inhibitors for copper in sulphuric acid," International Journal of Scientific Research, vol. 3, pp. 349-354, 2014.

[7] M. El Achouri, S. Kertit, M. Salem, M. Hajji, and E. Essassi, "Inhibitive effect of some 1,5-benzodiazepin 2-one derivatives on the corrosion of iron in $1 \mathrm{M} \mathrm{HCl,"} \mathrm{Journal} \mathrm{de} \mathrm{Chimie}$ Physique, vol. 93, pp. 2011-2024, 1996.

[8] K. Barouni, A. Kassale, A. Albourine, O. Jbara, B. Hammouti, and L. Bazzi, "Amino acids as corrosion inhibitors for copper in nitric acid medium: experimental and theoretical study," Journal of Materials and Environmental Science, vol. 5, pp. 456-463, 2014.

[9] A. M. Al-Fakih, Z. Y. Algamal, M. H. Lee, H. H. Abdallah, H. Maarof, and M. Aziz, "Quantitative structure-activity relationship model for prediction study of corrosion inhibition efficiency using two-stage sparse multiple linear regression," Journal of Chemometrics, vol. 30, no. 7, pp. 361-368, 2016.

[10] B. El Ibrahimi, A. Soumoue, A. Jmiai et al., "Computational study of some triazole derivatives (un- and protonated forms) and their copper complexes in corrosion inhibition process," Journal of Molecular Structure, vol. 1125, pp. 93-102, 2016.

[11] R. Kumar, H. Kim, and G. Singh, "Experimental and theoretical investigations of a newly synthesized azomethine compound as inhibitor for mild steel corrosion in aggressive media: a comprehensive study," Journal of Molecular Liquids, vol. 259, pp. 199-208, 2018.

[12] A. M. El Defrawy, M. Abdallah, and J. H. Al-Fahemi, "Electrochemical and theoretical investigation for some pyrazolone derivatives as inhibitors for the corrosion of C-steel in 0.5 M hydrochloric acid," Journal of Molecular Liquids, vol. 288, p. 110994, 2019.

[13] I. B. Obot, D. D. Macdonald, and Z. M. Gasem, "Density functional theory (DFT) as a powerful tool for designing new organic corrosion inhibitors. Part 1: an overview," Corrosion Science, vol. 99, pp. 1-30, 2015.

[14] H. Bourzi, R. Oukhrib, B. El Ibrahimi et al., "Furfural analogs as sustainable corrosion inhibitors-predictive efficiency using DFT and Monte Carlo simulations on the $\mathrm{Cu}(111), \mathrm{Fe}(110)$, $\mathrm{Al}(111)$ and $\mathrm{Sn}(111)$ surfaces in acid media," Sustainability, vol. 12 , no. 8 , p. $3304,2020$.

[15] B. El Ibrahimi, A. Jmiai, K. El Mouaden et al., "Theoretical evaluation of some $\alpha$-amino acids for corrosion inhibition of copper in acidic medium: DFT calculations, Monte Carlo simulations and QSPR studies," Journal of King Saud University Science, vol. 32, no. 1, pp. 163-171, 2020.

[16] H. Zhao, X. Zhang, L. Ji, H. Hu, and Q. Li, "Quantitative structure-activity relationship model for amino acids as corrosion inhibitors based on the support vector machine and molecular design," Corrosion Science, vol. 83, pp. 261-271, 2014.

[17] P. Lian, R. C. Johnston, J. M. Parks, and J. C. Smith, "Quantum chemical calculation of $\mathrm{pK}_{\mathrm{a}} \mathrm{s}$ of environmentally relevant functional groups: carboxylic acids, amines, and thiols in aqueous solution," The Journal of Physical Chemistry A, vol. 122, no. 17, 2018.

[18] P. F. B. Gonçalves and H. Stassen, "Calculation of the free energy of solvation from molecular dynamics simulations," Pure and Applied Chemistry, vol. 76, pp. 231-240, 2004.

[19] G. Duarte Ramos Matos, D. Y. Kyu, H. H. Loeffler, J. D. Chodera, M. R. Shirts, and D. L. Mobley, "Approaches for calculating solvation free energies and enthalpies demonstrated with an update of the FreeSolv database," Journal of Chemical \& Engineering Data, vol. 62, no. 5, pp. 1559-1569, 2017.

[20] G. Gece and S. Bilgiç, "A theoretical study on the inhibition efficiencies of some amino acids as corrosion inhibitors of nickel," Corrosion Science, vol. 52, no. 10, pp. 3435-3443, 2010.

[21] H. Gerengi, M. M. Solomon, S. Öztürk, A. Yıldırım, G. Gece, and E. Kaya, "Evaluation of the corrosion inhibiting efficacy of a newly synthesized nitrone against St37 steel corrosion in acidic medium: experimental and theoretical approaches," Materials Science and Engineering: C, vol. 93, pp. 539-553, 2018.

[22] N. Kovačević and A. Kokalj, “The relation between adsorption bonding and corrosion inhibition of azole molecules on copper," Corrosion Science, vol. 73, pp. 7-17, 2013.

[23] N. Kovačević and A. Kokalj, "Analysis of molecular electronic structure of imidazole- and benzimidazole-based inhibitors: a simple recipe for qualitative estimation of chemical hardness," Corrosion Science, vol. 53, pp. 909-921, 2011.

[24] Y. Qiang, S. Zhang, S. Xu, and W. Li, "Experimental and theoretical studies on the corrosion inhibition of copper by two indazole derivatives in $3.0 \% \mathrm{NaCl}$ solution," Journal of Colloid and Interface Science, vol. 472, pp. 52-59, 2016.

[25] M. S. Masoud, M. K. Awad, M. A. Shaker, and M. M. T. ElTahawy, "The role of structural chemistry in the inhibitive performance of some aminopyrimidines on the corrosion of steel," Corrosion Science, vol. 52, no. 7, pp. 2387-2396, 2010.

[26] S. Mukhopadhyay, S. Dasgupta, S. Roy et al., "Corrosion inhibition of mild steel by aqueous leaf extract of purple hedge plant: experimental and theoretical investigation," Journal of Bio- and Tribo-Corrosion, vol. 7, no. 4, p. 139, 2021.

[27] S. Hong, X. Bin, and Z. Hong-Jun, "Electrochemical and theoretical studies of 1-Alkyl-2-substituted Benzimidazoles as 
corrosion inhibitors for carbon steel surface in $\mathrm{HCl}$ Medium," Jiegou Huaxue, vol. 35, pp. 1829-1839, 2016.

[28] B. El Ibrahimi, "Atomic-scale investigation onto the inhibition process of three 1,5-benzodiazepin-2-one derivatives against iron corrosion in acidic environment," Colloid and Interface Science Communications, vol. 37, Article ID 100279, 2020.

[29] I. B. Obot, K. Haruna, and T. A. Saleh, "Atomistic simulation: a unique and powerful computational tool for corrosion inhibition research," Arabian Journal for Science and Engineering, vol. 44, no. 1, pp. 1-32, 2019.

[30] C. Verma, H. Lgaz, D. K. Verma, E. E. Ebenso, I. Bahadur, and M. A. Quraishi, "Molecular dynamics and Monte Carlo simulations as powerful tools for study of interfacial adsorption behavior of corrosion inhibitors in aqueous phase: a review," Journal of Molecular Liquids, vol. 260, pp. 99-120, 2018.

[31] B. El Ibrahimi, L. Bazzi, and S. El Issami, "The role of $\mathrm{pH}$ in corrosion inhibition of tin using the proline amino acid: theoretical and experimental investigations," RSC Advances, vol. 10, no. 50, Article ID 29696, 2020.

[32] C. D. Taylor, A. Chandra, J. Vera, and N. Sridhar, "A multiphysics perspective on mechanistic models for chemical corrosion inhibitor performance," Journal of the Electrochemical Society, vol. 162, no. 7, pp. C369-C375, 2015.

[33] P. Dohare, K. R. Ansari, M. A. Quraishi, and I. B. Obot, "Pyranpyrazole derivatives as novel corrosion inhibitors for mild steel useful for industrial pickling process: experimental and Quantum Chemical study," Journal of Industrial and Engineering Chemistry, vol. 52, pp. 197-210, 2017.

[34] B. El Ibrahimi, K. El Mouaden, A. Jmiai et al., "Understanding the influence of solution's $\mathrm{pH}$ on the corrosion of tin in saline solution containing functional amino acids using electrochemical techniques and molecular modeling," Surfaces and Interfaces, vol. 17, Article ID 100343, 2019.

[35] A. Fitoz, H. Nazır, M. Özgür, E. Emregül, and K. C. Emregül, "An experimental and theoretical approach towards understanding the inhibitive behavior of a nitrile substituted coumarin compound as an effective acidic media inhibitor," Corrosion Science, vol. 133, pp. 451-464, 2018.

[36] D. Kumar, N. Jain, V. Jain, and B. Rai, "Amino acids as copper corrosion inhibitors: a density functional theory approach," Applied Surface Science, vol. 514, Article ID 145905, 2020.

[37] L. H. Madkour, S. Kaya, L. Guo, and C. Kaya, "Quantum chemical calculations, molecular dynamic (MD) simulations and experimental studies of using some azo dyes as corrosion inhibitors for iron. Part 2: bis-azo dye derivatives," Journal of Molecular Structure, vol. 1163, pp. 397-417, 2018.

[38] A. Kokalj, "Is the analysis of molecular electronic structure of corrosion inhibitors sufficient to predict the trend of their inhibition performance," Electrochimica Acta, vol. 56, no. 2, pp. 745-755, 2010. 